\title{
Data-driven estimations of Standard Model backgrounds to SUSY searches in ATLAS
}

\author{
F. Legger on behalf of the ATLAS collaboration \\ Max-Planck-Institut für Physik, München, Germany
}

\begin{abstract}
At the Large Hadron Collider (LHC), the strategy for the observation of supersymmetry in the early days is mainly based on inclusive searches. Major backgrounds are constituted by mismeasured multi-jet events and $W, Z$ and $t$ quark production in association with jets. We describe recent work performed in the ATLAS Collaboration to derive these backgrounds from the first ATLAS data.
\end{abstract}

Keywords: SUSY, ATLAS, LHC, Standard Model, data-driven estimation

PACS: $29.85 . \mathrm{Fj}, 14.80 . \mathrm{Ly}$

\section{INTRODUCTION}

SUper SYmmetry (SUSY) is one of the most favourable scenarios for physics beyond the Standard Model (SM) [1,2]. With no evidence of SUSY found by experiments at LEP and so far at Tevatron, the LHC is the next candidate to observe it. Owing to the large number of SUSY models on the market, inclusive searches are to be preferred with the first LHC data. R-parity conserving SUSY models provide general event signatures such as several highly energetic jets, leptons, and large missing transverse energy $\left(E_{T, m i s s}\right)$ [3]. An excess of such events with respect to SM predictions will be a typical manifestation of SUSY. Due to insufficient knowledge of SM cross-sections, underlying event, parton showers, and parton distribution functions at LHC energies, as well as limited Monte Carlo statistics, a reliable estimation of the SM backgrounds should be extracted from collider data. In the ATLAS collaboration, several data-driven background estimation methods are currently under development [4]. The common strategy is the extrapolation of SM contributions from a control sample, with features similar to the signal sample but as free of SUSY events as possible. The control sample must allow for an unbiased prediction of SM background distributions, have low contamination from SUSY events or other SM backgrounds, but contain enough events to keep the statistical uncertainty low. In ATLAS, inclusive searches for SUSY are classified according to the number of required leptons $(0,1, \ldots)$. The main contributions to SM backgrounds in 0 -lepton mode SUSY searches are given by $t \bar{t}(67 \%), W(17 \%)$ and $Z(10 \%)$ with jets, and QCD events (10\%). The most dangerous SM background to 1-lepton mode searches is given by $t \bar{t}$ events (91\%). Other contributions come from $W(7 \%)$ and $Z(1 \%)$ with jets, and QCD events $(<1 \%)$. We present an overview of methods for the estimation of QCD, $Z$ and $t \bar{t}$ backgrounds with the ATLAS detector from early data $\left(1 \mathrm{fb}^{-1}\right.$ integrated luminosity is assumed in the following). We focus on the advantages and uncertainties of the methods, rather than on a detailed description of selection criteria, which can be found in [4]. 

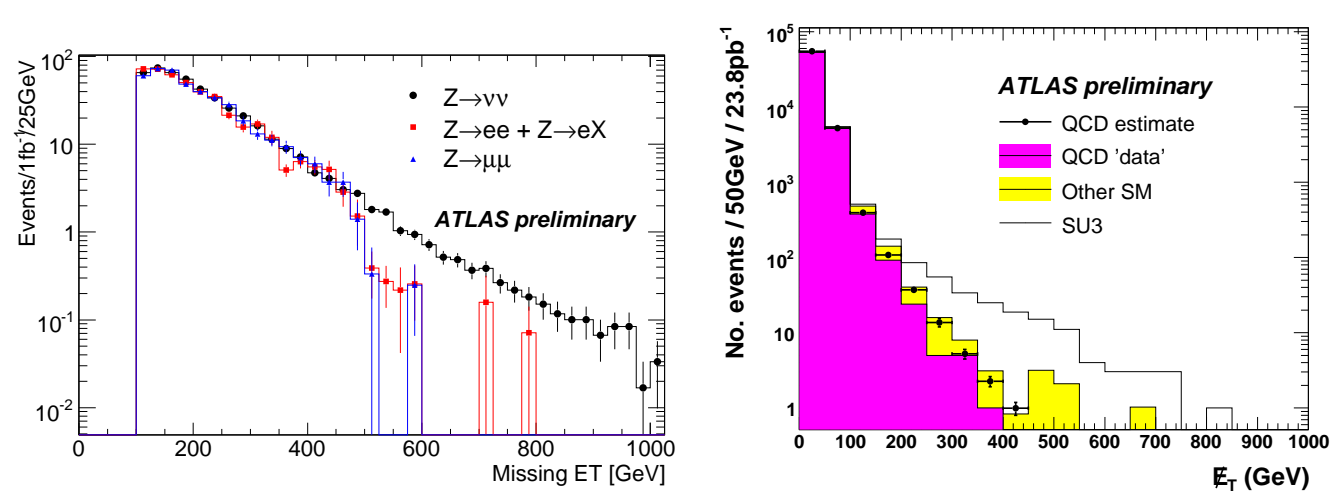

FIGURE 1. Missing transverse energy distibution of $Z \rightarrow v v$ (left) and QCD (right) events in 0-lepton mode SUSY searches. Data-driven estimations and Monte Carlo predictions are shown. Contributions from SUSY (SU3 benchmark point, defined in the minimal supergravity parameter space [5] by $m_{0}=100$ $\mathrm{GeV}, m_{1 / 2}=300 \mathrm{GeV}, A_{0}=-300 \mathrm{GeV}, \mu>0$ ) and other SM backgrounds are also shown with the QCD estimation.

\section{$Z \rightarrow v v$ ESTIMATION WITH REPLACEMENT METHOD}

The contribution of $Z \rightarrow v v$ events to SUSY searches with no lepton can be estimated from a control sample of $Z \rightarrow l l(l=e, \mu)$ events. A dilepton sample is selected by applying 0-mode-lepton SUSY selection and additionally requiring two leptons of the same flavour and opposite charge. The $E_{T, \text { miss }}$ is then replaced by $p_{T}(l l)$. The approximation that the main contribution to $E_{T, m i s s}$ in $Z \rightarrow v v$ events is given by the two neutrinos is justified by the ATLAS $E_{T, m i s s}$ resolution. To further reduce contaminations from other SM backgrounds or SUSY events, we require the invariant mass of the two leptons to be within $\pm 10 \mathrm{GeV}$ of the $Z$ mass, and $E_{T, \text { miss }}<30 \mathrm{GeV}$. After correcting for acceptance and lepton reconstruction efficiency, the estimation from the $Z \rightarrow l l$ sample agrees with the distribution of $Z \rightarrow v v$ events from Monte Carlo (see Fig. 1, left). However, due to the lower branching ratio of $Z \rightarrow l l$ with respect to $Z \rightarrow v v$, statistics of the control sample is limited in the high $E_{T, m i s s}$ region. This can be solved by using the shape from the Monte Carlo, and normalizing it to data, since studies show that the uncertainty in Monte Carlo predictions is coming mainly from the normalization, rather than from the shape. The systematic uncertainty on the number of estimated background events is $10 \div 15 \%$, mainly coming from the lepton identification efficiency and $E_{T, \text { miss }}$ scale. The statistic uncertainty is $\sim 13 \%$.

\section{QCD BACKGROUND ESTIMATION WITH JET SMEARING}

Large $E_{T, m i s s}$ values in multi-jet events may arise either from mismeasurement in the calorimeters, or from neutrinos emitted in semileptonic $b$ or $c$ decays. Both contributions can be estimated by measuring the calorimeter response function to jets in QCD events where $E_{T, \text { miss }}$ points in the direction of one of the jets. The jet response function is then used to smear the transverse momentum of jets in a control sample of multi-jet events 

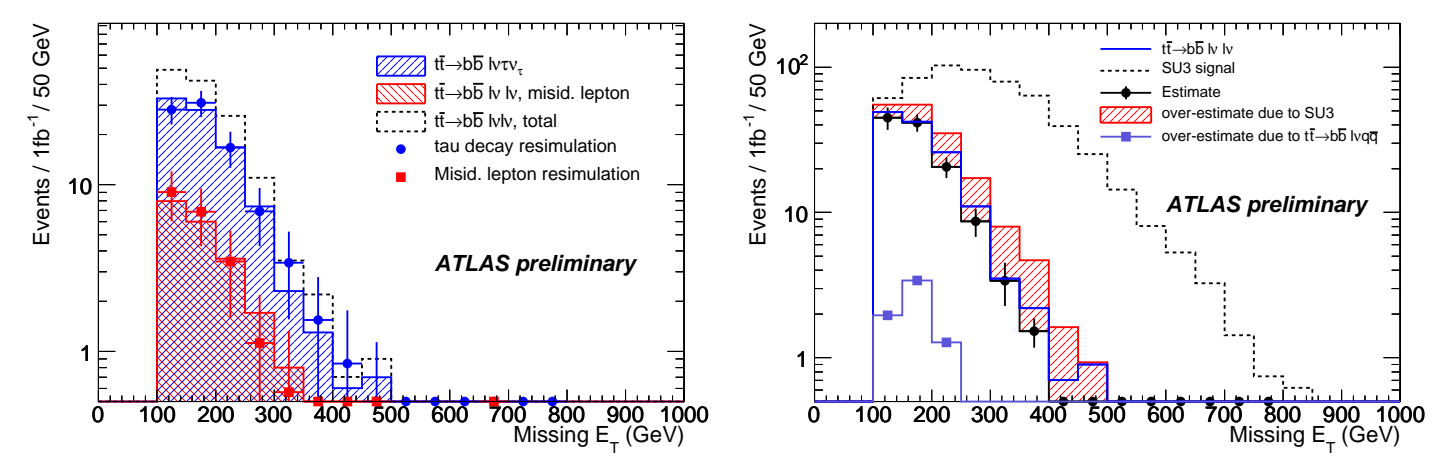

FIGURE 2. Missing transverse energy distibution of $t \bar{t} \rightarrow b \bar{b} l v l v$ events in 1-lepton mode SUSY searches. Contribution from events with a $\tau$ and misidentified leptons are shown on the left. On the right, contributions from SUSY and $t \bar{t} \rightarrow b \bar{b} l v q \bar{q}$ to the control sample are also shown.

with low $E_{T, m i s s}$. The estimated distribution of $E_{T, m i s s}$ in QCD multi-jet events is shown in Fig. 1 (right). The systematic uncertainty is $\sim 60 \%$, mainly due to the measurement of the jet response function. The SUSY contamination of the control sample is negligible due to the low $E_{T, m i s s}$ requirement. The statistic uncertainty is also low ( $\left.\sim 1 \%\right)$, due to the large cross-section of the control sample.

\section{$t \bar{t} \rightarrow b \bar{b} l v l v$ WITH KINEMATIC RECONSTRUCTION}

The most dangerous SM background to 1-lepton mode SUSY searches is given by $t \bar{t}$ events where both $W$ bosons decay leptonically, but where one lepton is missed because it is either a $\tau$ or not reconstructed. A control sample of clean $t \bar{t} \rightarrow b \bar{b} l v l v$ events is selected by requiring kinematic constraints specific to the $t \bar{t}$ decay. The contribution from events where the $W$ decays into a $\tau$ is estimated by replacing one of the lepton in the control sample with a $\tau$, and letting it decay with the TAUOLA package [6]. To estimate the contribution of misidentified leptons, one lepton of the control sample is removed, and all event variables are recalculated. After applying 1-lepton-mode SUSY search requirements and correcting for lepton identification efficiency, the contribution of $t \bar{t} \rightarrow b \bar{b} l v l v$ can be estimated by normalizing in the low $E_{T, \text { miss }}$ region. The systematic uncertainty is $\sim 20 \%$, mainly coming from the replacement and the normalization. The statistic uncertainty is $\sim 10 \%$. SUSY contamination to the control sample is sizable $(\sim 50 \%)$, due to the presence of two neutrinos which do not allow for a unambiguous reconstruction of the $t \bar{t}$ decay. The excess from the presence of SUSY is however larger than the over-estimation of the $t \bar{t}$ background (see Fig. 2).

\section{CONCLUSIONS}

A few methods under development in ATLAS for the data-driven estimation of SM backgrounds to SUSY searches have been presented. Predictions with $1 \mathrm{fb}^{-1}$ integrated luminosity are affected by systematic uncertainties ranging from 10 to $60 \%$, and statistic 
uncertainties of $\sim 10 \%$. The presence of SUSY will also affect the background estimations, however the over-estimation due to this effect is smaller than the SUSY signal itself.

\section{REFERENCES}

1. J. Wess, and B. Zumino, Nucl. Phys., B70, 39 (1974).

2. H. P. Nilles, Phys. Rep., 110, 1 (1984).

3. ATLAS collaboration, "ATLAS Detector and Physics Performance Technical Design Report", CERN/LHCC/99-15 (1999).

4. ATLAS collaboration, "Expected Performance of the ATLAS experiment, Detector, Trigger and Physics", CERN report (2008), in preparation.

5. L. Ibanez, Phys. Lett., 118B, 73 (1982).

6. S. Jadach et al., Comp. Phys. Comm., 76, 361 (1993). 